\title{
La imagen corporativa a través de una estrategia de marketing social en las empresas peruanas
}

\author{
Flor Angélica Lavanda Reyes \\ flavanda@unica.edu.pe \\ Universidad Nacional San Luis Gonzaga \\ Ica-Perú \\ Mario Gustavo Reyes Mejía \\ gustavo.reyes@unica.edu.pe \\ Universidad Nacional San Luis Gonzaga \\ Ica-Perú \\ Rosa Elvira Ruiz Reyes \\ rosa.ruiz@unica.edu.pe \\ Universidad Nacional San Luis Gonzaga \\ Ica-Perú \\ Luis Alberto Castillo Samanamud \\ luis.castillo@unica.edu.pe \\ Universidad Nacional San Luis Gonzaga \\ Ica-Perú
}

\section{RESUMEN}

Las empresas peruanas aplican estrategias de marketing con la finalidad de que los consumidores prefieran la marca de sus productos y servicios, así mismo demostrar su identificación con los problemas de la sociedad y su participación para solucionarlos. En la presente investigación el objetivo fue: Identificar de qué manera la imagen corporativa mejora a través de una estrategia de marketing social en las empresas peruanas. Entre Octubre 2021 y Febrero 2022 se analizó la entrega de mascarillas KN 95 que realizó el Grupo Económico Romero a través de su campaña “Detrás de una mascarilla siempre está lo más importante" con la finalidad de fomentar el uso y evitar contagios del Covid 19 en las familias peruanas. Se aplicó una encuesta virtual a nivel nacional de 08 preguntas, a una muestra formada por 460 participantes en noviembre del 2021. La información recolectada en el trabajo de campo demuestra los siguientes resultados significativos: el $83 \%$ de los encuestados expresó conocer esta campaña; el 78\% recibió y utilizó la mascarilla KN 95 y el 72\% mejoró su opinión sobre el Grupo Romero. Se concluye que, la imagen corporativa mejora significativamente a través de una estrategia de marketing social en las empresas peruanas.

Palabras claves: consumidor; covid 19; marketing social; imagen corporativa; posicionamiento. 


\title{
The corporate image through a strategy of social marketing in peruvian companies
}

\begin{abstract}
Peruvian companies apply marketing strategies so that consumers prefer the brand of their products and services, as well as demonstrate their identification with the problems of society and their participation in solving them. In this research, the objective was: to identify how the corporate image improves through a social marketing strategy in Peruvian companies. Between October 2021 and January 2022, the delivery of KN95 masks carried out by the Romero Economic Group was analyzed through its campaign "Behind a mask is always the most important" in order to promote the use and avoid contagion of Covid 19 in peruvian families. A nationwide virtual survey of 08 questions was applied to a simple of 460 participants in November 2021. The information collected in the field work shows the following significant results: $83 \%$ of the respondents said they knew about this campaign; 78\% received and used the KN95 mask and 72\% improved their opinión of the Romero Group. It is concluded that the corporate image improves significantly through a social marketing strategy in Peruvian companies.
\end{abstract}

Keywords: Consumer, Covid 19, social marketing, corporate image, positioning.

Artículo recibido: 15 enero 2022 Aceptado para publicación: 08 febrero 2022 Correspondencia: flavanda@unica.edu.pe Conflictos de Interés: Ninguna que declarar 


\section{1.- INTRODUCCION}

En los últimos años la opinión de los clientes sobre diversos temas económicos y sociales ha cambiado, sobre todo por la pandemia del Covid 19 que ha conscientizado a las personas acerca de los cambios bruscos que pueden presentarse y la reacción de diversos sectores de la sociedad ante ellos; estas acciones repercuten en la imagen que los consumidores tienen sobre estas empresas o instituciones, debido a que nuestro instinto expresa que en una situación de la comunidad todos deben participar en la solución.

Debido a ello las organizaciones tienen una posición en el mercado y es así como los clientes la identifican. Según Paul Hefting (1991) la imagen corporativa, es la personalidad de la empresa, es única, lo que la simboliza, dicha imagen tiene que estar impresa en todas las acciones, productos, servicios, etc. que involucren a la empresa para darle un mejor performance, de tal manera que se pueda repetir su imagen y posicionarla en su mercado objetivo para lograr mejores resultados.

También Gregory, J. (1999) explica que la identidad corporativa es el conjunto coordinado de signos visuales por medio de los cuales la opinión pública reconoce y memoriza a una entidad, un grupo o una institución. En ésta época de tanta competencia los consumidores fácilmente pueden olvidar una marca, por lo que la aplicación actual de estrategias de recordación son mucha más agresiva, directas y motivacionales, se utilizan colores, formas, imágenes y su propia canción para que los consumidores la relacionen, muchas organizaciones eligen un personaje reconocido para ser la imagen de la marca, de tal manera que aprovechan la identificación del personaje y la marca de la empresa.

Por otro lado, las empresas modernas asumen que tienen la responsabilidad y tarea fundamental de concientizar a la población sobre elementos que mejoren su calidad de vida. Una herramienta indispensable para lograrlo es el marketing social definido por Kotler (2013) como un conjunto de estrategias de marketing enfocadas en generar valor para los clientes, de tal manera que se garantice el bienestar de éstos y de la comunidad en general, volviendo a los ciudadanos en consumidores de bienes sociales. Se requiere que los jóvenes encuentren valores, historia, costumbres que identifiquen al país de una manera más dinámica, por lo que las empresas eligen resaltarlos a través de campañas publicitarias, promocionales, que den buenos resultados. Por ejemplo, la Empresa Donofrio lanzó al mercado su producto estrella llamado chocolate sublime, con una 
envoltura de llamativos colores, que resaltan los carnavales en Perú, una fiesta tradicional que se celebra en diversos pueblos y cada uno tiene características particulares.

El Grupo Romero y su Fundación con el objetivo de ayudar a proteger a las familias peruanas y evitar más contagios por el Covid 19, así como para demostrar su compromiso con los ciudadanos, lanzó la Campaña "Detrás de una mascarilla, está lo más importante", quienes en alianza con los diarios de mayor circulación en el país El comercio, Perú 21, Trome y Correo, entregaron en Octubre 2020 un millón de mascarillas KN 95 en un empaque sanitario individual complemente sellado, colocado en una caja que garantiza su protección e inocuidad durante el proceso de distribución.

Debido a la gran acogida de la campaña y por su compromiso social, la Fundación Romero realiza en Mayo 2021 la segunda entrega de un millón de mascarillas KN95 en la misma modalidad, debido a la gran cantidad de contagios que se presentaron en la segunda ola de la pandemia.

En Enero de 2022 la Fundación Romero lanzó la Campaña “Cuidémonos juntos como en familia" donde se entregaron dos millones de mascarillas para contrarrestar la tercera ola, sumando así la entrega de cuatro millones de mascarillas gratis a la población peruana, que al comprar el diario recibe una caja donde además de la mascarilla recibe un mensaje de aliento y motivación para cuidarse y cuidar a su familia.

La presente investigación tiene por objetivo Identificar de qué manera la imagen corporativa mejora a través de una estrategia de marketing social en las empresas peruanas, analizando las campañas realizadas por el Grupo Romero y la respuesta de los consumidores, los cuales tienen un concepto percibido de la misma.

\section{2.- MATERIALES Y METODOS}

En la investigación se utilizó una metodología cuantitativa, de diseño no experimental del tipo descriptivo de corte transversal. La población materia de estudio estuvo conformada por consumidores de diferentes ciudades del Perú.

El trabajo de campo se realizó a nivel nacional a una muestra de 460 participantes en Enero 2022 utilizando medios digitales, tales como: email y redes sociales debido a las restricciones por el Covid 19 vigentes en el país. La investigación se desarrolló entre Octubre 2021 y Febrero 2022. La técnica aplicada fue la encuesta, elinstrumento utilizado fue un cuestionario de 08 preguntas, de tipo abierta, cerrada y de opción múltiple, que nos permitió obtener información acerca de las variables e indicadores de estudio: imagen 
corporativa y marketing social. Se utilizó la estadística descriptiva para el proceso y presentación de los resultados a través de cuadros y gráficos. El análisis de la información se realizó de manera cualitativa en relación a los objetivos e hipótesis propuestos.

\section{3.- RESULTADOS Y DISCUSION}

Tabla $\mathbf{N}^{\circ}$ 1. Imagen corporativa

\begin{tabular}{|l|c|c|}
\hline & $\begin{array}{c}\text { ¿Generalmente tiene ud una opinión sobre las } \\
\text { empresas peruanas? }\end{array}$ & $\%$ \\
\hline Siempre & 160 & 35 \\
\hline Casi Siempre & 120 & 26 \\
\hline Regularmente & 100 & 22 \\
\hline Muy pocas veces & 80 & 17 \\
\hline TOTAL & $\mathbf{4 6 0}$ & $\mathbf{1 0 0}$ \\
\hline
\end{tabular}

Fuente. Elaboración propia.

El 35\% de los encuestados expresó que siempre tiene una opinión sobre las empresas peruanas, por las diversas marcas de productos que comercializan, por las actividades que realizan en la comunidad y porque los clientes comentan en las redes sociales sobre el servicio que han recibido, también hay opiniones de los trabajadores de las empresas que comentan si es un buen lugar para trabajar, todo ello hace que la sociedad se forme una opinión sobre las organizaciones.

Según Riordan, Gatewood y Barnes Bill (1997), la Imagen corporativa es la percepción individual de las acciones, actividades y compromisos que demuestra una organización; asimismo, es el dibujo mental que una audiencia tiene sobre la empresa, como consecuencia de los diversos mensajes que ha recibido, por ejemplo: un el cliente compra un producto y luego decide cambiarlo, cuando va a la empresa lo atienden con amabilidad y hacen el cambio por otro producto, el mensaje recibido será favorable por lo que el recuerdo mental será duradero.

Según Capriotti (2013): La Imagen Corporativa es la imagen que tienen los públicos de una organización en cuanto entidad. Es la idea global que tienen sobre sus productos, sus actividades y su conducta”, lo que implica cuidar cada detalle de lo que ofrece, porque se relacionan con las necesidades y deseos de su público objetivo, el cual tiene una expectativa que al ser cumplida tendrá un grado de satisfacción mayor con la empresa y sus comentarios serán favorables. 
Según Dutton y Dukerich (1991), existe una imagen interna y una externa que se relacionan, por lo que la existencia de una discrepancia entre lo que los miembros de la organización piensan de ella (identidad organizacional) y lo que creen que los demás piensan de la organización (imagen corporativa) es el desencadenante del proceso de reflejo, es decir, lo que piensan y sienten los empleados por su organización se refleja en la calidad de los productos que elabora, en el servicio y atención amable que ofrece a sus clientes, etc. los primeros clientes satisfechos deberán ser los empleados.

Tabla $\mathbf{N}^{\circ}$ 2. Imagen Positiva

\begin{tabular}{|l|c|c|}
\hline & $\begin{array}{c}\text { Considera ud. que las empresas se preocupan por } \\
\text { tener una imagen positiva }\end{array}$ & $\%$ \\
\hline Siempre & 200 & 44 \\
\hline Casi siempre & 160 & 34 \\
\hline Regularmente & 100 & 22 \\
\hline Nunca & 0 & 0 \\
\hline TOTAL & $\mathbf{4 6 0}$ & $\mathbf{1 0 0}$ \\
\hline
\end{tabular}

Fuente. Elaboración propia.

El 44\% de los encuestados considera que siempre las empresas se preocupan por tener una imagen positiva, porque su principal objetivo es mantener satisfechos a los clientes, debido a ello buscan ser líderes en el mercado y posicionarse a través de promociones de venta, publicidad, auspicios y patrocinios en favor de la comunidad.

Según Ramos, E. (2020) menciona que en investigaciones realizadas por Patlán Pérez \& Martínez (2017), se demostró que la imagen organizacional es significativa para muchas organizaciones debido a que en la actualidad nos encontramos en un ambiente considerablemente competitivo, donde cada organización tiene diferencias significativas de su imagen. Es decir, para permanecer en el mercado, las empresas deben realizar marcadas diferencias en sus productos o servicios que sean fáciles de recordar por los clientes y les haga tener un concepto favorable de ellas, por la gran cantidad de empresas que compiten por ganar la preferencia de los consumidores.

Según Costa (1995) la imagen corporativa puede ser positiva, cuando los clientes la recuerdan fácilmente porque han logrado posicionarme por el precio más bajo, menor tiempo de espera en la atención, variedad en los productos, etc, pero existen empresas que no logran que su imagen sea positiva, debido a que no envían a tiempo el pedido, 
cambian constantemente de número telefónico, no brindan servicio delivery, etc, y todo ello constituye una imagen negativa en los consumidores, los cuales difunden su descontento haciendo cada vez mayor el desinterés.

Tabla No 3. Temas Sociales

\begin{tabular}{|l|c|c|}
\hline & $\begin{array}{c}\text { Considera ud. que las empresas deben educar a } \\
\text { los consumidores sobre temas sociales }\end{array}$ & $\mathbf{\%}$ \\
\hline SI & 360 & 78 \\
\hline NO & 100 & 22 \\
\hline TOTAL & $\mathbf{4 6 0}$ & $\mathbf{1 0 0}$ \\
\hline
\end{tabular}

Fuente. Elaboración propia.

El 78\% de los encuestados considera que las empresas SI deben educar a los consumidores sobre temas sociales, porque están en contacto directo con ellos y ejercen un nivel de influencia significativo; asimismo, las empresas son parte de la sociedad y son afectados por todos los problemas de su comunidad y al tener una identificación con los ciudadanos ellos la consideraran de una mejor manera.

Según Beall y otros (2012) el marketing social es una tendencia que ha generado cambios favorables en la sociedad y ha brindado múltiples beneficios a las organizaciones que han emprendido iniciativas sociales, por lo que las empresas en los envases de sus productos anotan consejos para la tener una vida más saludable, además de constituir oportunidades de innovación empresarial para transformar la sociedad.

Según French J. y Gordon R. (2015) El marketing social tiene como objetivo fundamental incidir en el comportamiento y la conducta deseados de personas y grupos en un entorno marcado por la complejidad política, económica y social. En las condiciones de pandemia por el Covid 19 los individuos requieren sentirse acompañados en este proceso, así como que otras personas o empresas sientan empatía por lo que está ocurriendo.

Según Kotler P., Roberto E. (1992) expresan que las empresas piensan en el producto social, como aquel que va satisfacer necesidades insatisfechas, por ejemplo, la empresa Colgate-Palmolive Company, lanzó al mercado el spray antibacterial para manos, que elimina el $99 \%$ de bacterias para cuidado contra el covid 19 en respuesta a la campaña del lavado de manos, lo que fomenta un cambio de conducta en los ciudadanos al tener una alternativa en cualquier lugar. 
Tabla No 4. Mascarillas KN 95

\begin{tabular}{|c|c|c|}
\hline & $\begin{array}{c}\text { ¿Cuántas mascarillas KN 95 utiliza ud. en } \\
\text { promedio al mes? }\end{array}$ & \% \\
\hline Entre 3 y 5 unid & 160 & 35 \\
\hline Entre 6 y 9 unid & 100 & 22 \\
\hline Más de 10 unid & 60 & 13 \\
\hline No utiliza KN95 & 140 & 30 \\
\hline TOTAL & $\mathbf{4 6 0}$ & $\mathbf{1 0 0}$ \\
\hline
\end{tabular}

Fuente. Elaboración propia.

El 35\% de los encuestados expresó utilizar entre 3 y 5 mascarillas KN95 en promedio al mes, debido a que utiliza dos mascarillas, es decir, en la parte exterior coloca una desechable quirúrgica, la cual puede cambiar diariamente debido a que es más económica y las recibe en su trabajo mensualmente de manera gratuita.

El 30\% de los encuestados expresó no utilizar la mascarilla KN95 debido a que son muy caras y su presupuesto no le permite comprarla, a diferencia de las desechables que puede adquirirlas con mayor facilidad y en muchos casos se la entregan en su trabajo, es consciente que la calidad y seguridad que brindan este tipo de mascarillas es mejor y desearía utilizarlas.

Según Olivera E. (2018) en las conclusiones de su investigación, expresa que la finalidad primordial del marketing social es contribuir con el bienestar y mejora de la calidad de vida de los miembros de la sociedad en su conjunto, por lo que observa cuidadosamente los problemas que pueden resolverse a corto plazo.

Tabla N 5. Campaña Grupo Romero

\begin{tabular}{|l|c|c|}
\hline & $\begin{array}{c}\text { ¿Conoce ud. la campaña de entrega de } \\
\text { mascarillas KN95 por el Grupo Romero? }\end{array}$ & $\mathbf{\%}$ \\
\hline SI & 380 & 83 \\
\hline NO & 80 & 17 \\
\hline TOTAL & $\mathbf{4 6 0}$ & $\mathbf{1 0 0}$ \\
\hline
\end{tabular}

Fuente. Elaboración propia.

El $\mathbf{8 3 \%}$ de los encuestados expresó que, SI conoce la campaña de entrega de mascarillas KN95 por el Grupo Romero, debido a que han utilizado una buena publicidad en diversos medios y las reparten con diarios de gran circulación a nivel nacional. Otro aspecto que le parece interesante, es que una empresa se preocupe por entregar ese tipo de mascarillas que tienen un alto precio en el mercado y no están al alcance de la mayoría, y en las tres olas de pandemia Covid 19 que se han presentado en el país hasta la fecha. 
Según Kotler y Armstrong (2013) consideran que las empresas aplican marketing social como un conjunto de estrategias enfocadas en generar valor para los clientes, para su bienestar y el de la comunidad, volviendo a los ciudadanos en consumidores de bienes sociales. A lo que Mitchell, Madill y Chreim (2015) agregan que todas las empresas sociales buscan transformar la sociedad, y lo único que varían son los métodos para lograrlo, es decir, unos más creativos, innovadores, directos, etc. que otros y el tiempo en que se aplican unos obtendrán resultados a corto plazo y otros a mediano plazo.

Según Fernández A. (2017) en su artículo menciona a Briceño, S. (2010) considera que la gestión del marketing social se aplica en varias etapas, considerando el análisis de las variables internas y externas con relación al producto o servicio social, tomando como variable indispensable los medios que serán utilizados para persuadir, motivar e influir en la decisión del consumidor a través de la publicidad, la promoción, el marketing directo, el patrocinio y otros medios.

Los autores key y Czaplewski (2017) explican las cinco etapas que desarrolla una estrategia de marketing social:

1. Determinación del público objetivo: Se selecciona un público específico considerando todas aquellas externalidades que se puedan presentar.

2. Delimitación del público objetivo periférico: Requiere un conocimiento profundo del público objetivo.

3. Selección del canal adecuado: escoger las mejores alternativas que permitan el flujo de comunicación entre las organizaciones y el público.

4. Generación del mensaje: El mensaje que se va a transmitir al público objetivo debe ser comprensible, claro y preciso.

5. Control: Se requiere de la retroalimentación para poder medir los resultados y adoptar medidas correctivas en caso de inconvenientes.

Tabla $N^{\circ}$ 6. Diarios de la campaña

\begin{tabular}{|l|c|c|}
\hline & $\begin{array}{c}\text { iAdquirió ud. los diarios que entregaban las } \\
\text { mascarillas KN95 del Grupo Romero? }\end{array}$ & $\%$ \\
\hline Siempre & 220 & 48 \\
\hline Casi siempre & 140 & 30 \\
\hline Regularmente & 100 & 22 \\
\hline Nunca & 0 & 0 \\
\hline TOTAL & $\mathbf{4 6 0}$ & $\mathbf{1 0 0}$ \\
\hline
\end{tabular}

Fuente. Elaboración propia. 
El $\mathbf{4 8 \%}$ de los encuestados expresó que siempre y el $\mathbf{3 0 \%}$ casi siempre, lo que significa el 78\% de la muestra, adquirió los diarios: El comercio, Perú 21, Trome o Correo, que entregaban las mascarillas KN95 del Grupo Romero. Cada diario está dirigido a un segmento de mercado diferente por el precio y tipo de información que ofrecen, lo que aseguraba que pudieran llegar a diversos tipos de público.

La entrega se organizó durante fechas que fueron programadas para cada diario, durante los cuatro fines de semana de Octubre 2020, Mayo 2021 y Enero 2022, porque consideraba una excelente oportunidad de adquirir el diario y obtener una mascarilla de ese tipo por el mismo precio, cuando su valor era superior al adquirirla sola en una farmacia.

Los consumidores han expresado también que felicitan al Grupo Romero y el Grupo El Comercio, los cuales se unieron para facilitar a los ciudadanos peruanos el cuidado y la protección necesaria en plena pandemia del Covid 19, cuando la economía de las familias estaba deteriorada y muchos de ellos no tenían acceso a ningún tipo de cuidado.

Según García Uceda, M. (2011) resalta la importancia de la comunicación entre empresa y consumidor, así como la elección de los medios publicitarios que son los vehículos por excelencia para dar a conocer y difundir una noticia, promocionar ideas, productos o servicios, los cuales pueden ser convencionales o alternativos.

Según Sánchez Calderón (2009) el periódico es un medio de comunicación escrito y publicitario. Su costo en publicidad varía según ubicación, tamaño, color y tipo de anuncio que se desea dar a conocer y la frecuencia con que aparecerá el anuncio. Tiene como Ventajas: una amplia penetración, flexibilidad, selectividad geográfica, involucramiento del lector, servicios especiales, y poder insertar material publicitario pagado.

Tabla No $\mathbf{N}^{\circ}$ Opinión de la campaña

\begin{tabular}{|l|c|c|}
\hline & $\begin{array}{c}\text { Considera ud. que la campaña de entrega de } \\
\text { mascarillas KN95 por el Grupo Romero fue: }\end{array}$ & $\%$ \\
\hline Muy Buena & 240 & 52 \\
\hline Buena & 160 & 35 \\
\hline Regular & 60 & 13 \\
\hline Mala & 0 & 0 \\
\hline TOTAL & $\mathbf{4 6 0}$ & $\mathbf{1 0 0}$ \\
\hline
\end{tabular}

Fuente. Elaboración propia. 
El 52\% de los encuestados considera muy buena la campaña de entrega de mascarillas KN 95 por el Grupo Romero, porque se han beneficiado cuatro millones de personas de diversas zonas del país, muchos de los cuales no tenían a su alcance este tipo de protección; también considera que la entrega en una caja con protección del producto y el mensaje que motivaba a la adquisición de los diarios por las mascarillas.

Según Giuliani, A. (2012) el Marketing vinculado a una causa social se asocia a la existencia de una relación directa entre una determinada actividad de consumo y una donación realizada por la empresa a aquella causa.

La Campaña "Detrás de una mascarilla, está lo más importante" también tuvo como beneficiarios a diez mil canillitas (vendedores de diarios que se desplazan de un lugar a otro para vender sus periódicos) de todo el Perú, por considerarlos elemento indispensable en la entrega de las mascarillas a la sociedad y para brindarles una mayor protección por la exposición diaria de su trabajo. Otro grupo beneficiado fueron los bomberos del país, los cuales realizan su labor de manera gratuita por su vocación de servicio y ayuda a la comunidad, por lo que doscientos mil recibieron mascarillas KN 95 como parte de esta campaña del Grupo Romero.

Esta campaña de gran éxito en el Perú, seguirá vigente debido a que durante los próximos seis meses los beneficiarios serán los pacientes de cáncer del Instituto Nacional de Enfermedades Neoplásicas (INEN), quienes a través de la Fundación Peruana de Cáncer serán los encargados de entregarlas a los pacientes que acudan a su tratamiento médico.

Tabla $\mathbf{N}^{\mathbf{0}} 8$. Imagen corporativa del Grupo Romero

\begin{tabular}{|l|c|c|}
\hline & $\begin{array}{c}\text { Considera ud. que la imagen corporativa del } \\
\text { Grupo Romero después de entrega de } \\
\text { mascarillas KN95 mejoró en: }\end{array}$ & $\%$ \\
\hline Credibilidad & 80 & 18 \\
\hline Genera confianza & 110 & 24 \\
\hline Empatía con la Sociedad & 140 & 30 \\
\hline No mejoró & 130 & 28 \\
\hline TOTAL & $\mathbf{4 6 0}$ & $\mathbf{1 0 0}$ \\
\hline
\end{tabular}

Fuente. Elaboración propia.

El 30\% de los encuestados considera que la imagen corporativa del Grupo Romero mejoró después de la entrega de mascarillas KN 95, porque la empresa demostró tener empatía con la sociedad, debido a la gran cantidad de contagios que se presentaban en el 
país por el Covid 19 sobretodo en los de menos recursos económicos que debían exponerse al salir a trabajar y estar en contacto con muchas personas.

Según He, H. y Balmer (2007) expresaron que a nivel corporativo hay tres conceptos que deben considerarse para el análisis de la opinión interna y externa de los clientes, tales como imagen, reputación y comunicación corporativa. A lo que Dutton y Dukerich (1991) complementan que las organizaciones no tienen poder suficiente para determinar directamente su imagen pública ni su reputación, pero puede influir en su formación a través de una gestión adecuada de la identidad de la empresa y es a través de diversas campañas de marketing social, auspicios, responsabilidad social, puede hacer que los consumidores lo prefieran y mejoren su opinión sobre todo en este contexto digital donde los clientes comparten comentarios sobre los productos y servicios que adquieren. Finalmente, Karaosmanoglu y Melewar (2006), sugieren que la imagen y reputación de una empresa se forman a partir de la proyección del conjunto de signos que conforman su identidad.

Según Pérez A. (2014) en su investigación menciona a Walker (2010), quien expresa que la imagen organizacional, hace referencia bien a la imagen que la empresa desea proyectar hacia sus grupos de interés (imagen deseada ) o a la imagen real que estos individuos tienen sobre la empresa (imagen real), por lo que hay una diferencia es estos dos aspectos, porque la imagen real puede no ser igual a la deseada por la empresa, en el caso contrario puede obtenerse resultados más favorables de lo deseado y lograr posicionarme en el mercado de una mejor manera, por la aplicación de una estrategia que resultó favorable para la sociedad.

\section{4.- CONSIDERACIONES FINALES}

La imagen corporativa significa un elemento importante tanto dentro como fuera de las empresas, porque ello influye en la opinión que los consumidores tengan de ellas. Por lo que aplican diversas estrategias de marketing para posicionarse en la mente de los clientes como la empresa más económica, más responsable, con mayor garantía, etc.

El Grupo Económico Romero lanzó al mercado una estrategia de marketing social, una campaña para motivar a los consumidores el uso y la protección de la mascarilla en la epidemia de Covid 19 en Perú, teniendo un gran éxito y aceptación, por lo que fue realizada en tres oportunidades (años 2020; 2021 y 2022).

Esta campaña consiguió mejorar la opinión del Grupo Romero en la sociedad peruana, 
logró también la fidelización de los clientes durante las tres oportunidades que entregó las mascarillas, por lo que concluimos que: la imagen corporativa mejora significativamente a través de una estrategia de marketing social en las empresas peruanas.

\section{5.- LISTA DE REFERENCIAS}

Beall, T., Wayman, J., D’Agostino, H., Liang, A., \& Perellis, C. (2012). Social marketing at a critical turning point. Journal of Social Marketing. Vol.2. pp. 103117. DOI: $10.1108 / 20426761211243946$.

Briceño S, Mejías I, Godoy E. (2010): Comunicación de Marketing. International Journal of Good Conscience. Vol. 5. pp.98-113.

Capriotti, P. (2013). Planificación Estratégica de la Imagen Corporativa. Santiago: Andrós Impresores.

Costa, Joan (1995): Comunicación corporativa y revolución de los servicios. Madrid: Ciencias sociales.

Dutton, J. y J. Dukerich (1991). "Keeping an Eye on the Mirror: Image and Identity in Organizational Adaptation”. Academy of Management Journal, 34 (3), pp. 517 554.

Fernández, A.; Pérez, C.; Méndez, V.; Fernández, C.; Méndez, A. y Calero, S. (2017) Marketing Social y su influencia en la solución de problemas de salud. Revista Cubana de Investigaciones Biomédicas. Vol.36 N 3. Cuba. Consultado en: http://scielo.sld.cu/scielo.php?script=sci_arttext\&pid=S0864030020170003000 $\underline{11}$

French J, Gordon R. Strategic Social Marketing Sidney: Macquarie University; 2015.

Fundación Romero (2021): Artículo Entrega de un millón de mascarillas KN95 https://www.fundacionromero.org.pe/prensa/grupo-romero-entrega-otra-vez-1millon-de-mascarillas-kn95

Fundación Romero (2022): Artículo Grupo Romero vuelve a entregar dos millones de mascarillas

KN95

https://www.fundacionromero.org.pe/detrasdeunamascarilla/fundacionromero-vuelve-entregar-2-millones-de-mascarillas-kn95

García Uceda, M. (2011). Las claves de la publicidad. España: Esic Editorial. Séptima Edición. 
Giuliani A.; Monteiro, T.; Zambón, M.; Betanho, C.; Lima, L. (2012). Las claves de la publicidad. España: Esic Editorial. Séptima Edición. El Marketing Social, relacionado con causas sociales y la Responsabilidad Social Empresarial : El caso del Supermercado PÃO DE AÇÚCAR, de Brasil. Revista Invenio. Vol. 15. Núm. 29. pp. 11-27 Universidad del Centro Educativo Latinoamericano. Rosario -Argentina. Consultado en: https://www.redalyc.org/pdf/877/87724146003.pdf

Gregory J. y Weichmann (1999): Libro Marketing Corporate Image: The Company as your number one product. Editorial Mc Graw Hill

He, H. y Balmer, J.M.T., 2007. Identity studies: Multiple perspectives and implications for corporatelevel marketing. European Journal of Marketing, Vol.41. Núm.7/8 pp. 765-785.

Hefting, P. (2004). Manual de imagen corporativa. Editorial Gustavo Gili. Barcelona

Karaosmanoglu, E. y Melewar, T.C., 2006. Corporate communications, identity and image: A research agenda. Journal of Brand Management. Vol.14. pp. 196-206.

Key, T., \& Czaplewski, A. (2017). Upstream social marketing strategy: An integrated marketing communications approach. Business Horizons. Vol.60 Núm.3. Pp.325-333. DOI: 10.1016/j.bushor.2017.01.006.

Kotler Philip, Armastrong Gary (2013): Fundamentos de Mercadotecnia. Editorial Prentice Hall -12 ava Edición

Kotler P, Roberto E. Marketing Social (1992): Estrategias para cambiar la conducta pública. Madrid: Ediciones Díaz de Santos S.A.

Mitchell, A., Madill, J., \& Chreim, S. (2015). Marketing and social enterprises: implications for social marketing. Journal of Social Marketing. Vol.5. Núm.4. pp. 285-306. DOI: 10.1108/JSOCM-09-2014-0068.

Olivera, E., Pulido, V. (2018): Marketing Social: Su importancia en la resolución de problemas sociales. Revista Científica de la UCSA. Vol. 5 Núm.2 Paraguay. Consultado

en: http://scielo.iics.una.py/scielo.php?script=sci_arttext\&pid=S240987522018000 $\underline{200026}$

Patlán Pérez, J. y Martínez E. (2017): Evaluación de la imagen organizacional universitaria en una institución de educación superior. Revista Contaduría y 
Administración. Vol. 62 pp. 105-122 Núm.2. México. Consultado en: http://www.scielo.org.mx/pdf/cya/v62n1/0186-1042-cya-62-01-00105.pdf

Pérez, A.; Rodríguez del Bosque, I. (2014): Identidad, imagen y reputación de la empresa: integración de propuestas teóricas para una gestión exitosa. Cuadernos de Gestión, Vol. 14. Núm. 1. pp. 97-126 Universidad del País Vasco/Euskal Herriko Unibertsitatea Vizcaya, España. Disponible en: http://www.redalyc.org/articulo.oa?id=274330593005

Ramos, E.; Valle, N. (2020): Gestión de imagen corporativa como estrategia de sostenibilidad: camino al cambio empresarial. Revista Universidad y Sociedad. Vol.12 $\mathrm{N}^{\circ} 1$ Cuba. Consultado http://SCIELO.SLD.CU/SCIELO.PHP?SCRIPT=SCI_ARTTEXT\&PID=S221 $\underline{8-36202020000100292}$

Riordan, C.M., R.D. Gatewood y J. Barnes Bill (1997). Corporate Image: Employee Reactions and Implications for Managing Corporate Social Performanceî. Journal of Business Ethics, Vol.16. pp. 401-412.

Sánchez Calderón, H. (2009). Guía de Planificación Básica de Medios. Quito, Ecuador.

Walker, K. (2010). A Systematic review of the corporate reputation literature:

Definition, measurement, and theory. Corporate Reputation Review, Vol.12. Núm.4. pp. 357-387. 